\title{
An Underwater Sensor Network with Dual Communications, Sensing, and Mobility
}

\author{
Carrick Detweiller Iuliu Vasilescu Daniela Rus \\ Computer Science and Artificial Intelligence Laboratory \\ Massachusetts Institute of Technology \\ Cambridge, 02139 \\ Email: groups.csail.mit.edu/drl
}

\begin{abstract}
This paper describes an underwater sensor network with dual communication and support for sensing and mobility. The nodes in the system are connected acoustically for broadcast communication using an acoustic modem we developed. The nodes are connected optically for higher speed point to point data transfers using an optical modem we developed. We describe the hardware details of the underwater sensor node and the communication and networking protocols. Finally, we present and discuss the results from experiments with this system.
\end{abstract}

\section{INTRODUCTION}

Underwater modeling, mapping, and monitoring for marine biology, environmental, and security purposes are currently done manually or using expensive hard to maneuver underwater vehicles or individual instruments. We would like to bring a new level of automation and capability to this domain in the form of versatile and easily deployable underwater sensor networks. Just like the Berkeley Mica Mote [11], the current industry standard for ground sensor networks has fueled an explosion in the development of ground and aerial sensor network applications, our goal is to develop underwater technology that will enable a similar level of automation.

More than $70 \%$ of our planet is covered by water. It is widely believed that the underwater world holds ideas and resources that will fuel much of the next generation of science and business. However, any underwater operations are fraught with difficulty due to the absence of an easy way to collect and monitor data. Underwater sensors exist but they are not networked and their use has many issues:

- Deploying, retrieving, and using the sensors is labor intensive;
- Collecting the data is subject to very long delays;

- The manual aspects of using the sensors leads to error;

- The spatial scope for data collection with individual sensor is limited;

- Individual sensors are unable to perform operations that require cooperation, such as tracking relative movement and locating events.

What is required is a low-cost, versatile, highquality, easily deployable, self-configurable platform for underwater sensor networks that will (a) automate data collection and scale-up in time and space, (b) speed-up access to the collected data, and (c) be easy to use.

In this paper we describe the underwater sensor network hardware we designed, built, and deployed in lakes, rivers, and ocean. The hardware consists of static sensor network nodes and mobile robots that are dually networked optically (for point-topoint transmission at $330 \mathrm{~kb} / \mathrm{s}$ and acoustically for broadcast communication over hundreds of meters range at $300 \mathrm{~b} / \mathrm{s}$ ). We discuss the communication performance of the network during experiments with this system in the ocean, in rivers, and in lakes. We describe the sensor network hardware, explain the communication protocols, and show results from field experiments.

The sensor nodes, which were developed in our lab, are shown in Figure 1. These nodes package communication, sensing, and computation in a small cylindrical water-tight container. Each unit includes an acoustic modem and an optical modem implemented using green light and designed in our lab. The system of sensor nodes communicates with a TDMA protocol and is self-synchronizing. The system is 
capable of ranging and has a data rate of $300 \mathrm{~b} / \mathrm{s}$ verified up to 400 meters in fiesh water and in the ocean. The sensors in the unit include tercperature, pressine, and carcera with irquts for water chemistry sensous.

Because the nodes are light and small, they are easily deployed by marazlly throwing them overboard once deployed the nodes ane anchored with weights and form a static underwater retwork. This network self-localizes using a range based $3 D$ distributed boslization algorithon extersion of the $2 \mathrm{D}$ distributed localization algorithon devebped in our previous work [13].

\section{A. Related Woris}

There has been a growing interest in automating oceanographic researoh applications. This researh is motivated by the vision of collaborative oceanographis resean projects such as the Autorcomons Ocean Sarmpling Network II [1] and [2], [5], [6], [10]. It is becorning roore irmortant for robots and sensing irstruments to be able to assist in the deployment of measuring systems or to act as part of large-scale dats-collecting netwooks.

In designing the urderwater retwork system, we drow from important results in acoustic telemetry [4] and the design of sersor networks for werial operations [11]. A cabled water operation system was desaribed in [12]. Whe aloo build on the success of the WHOI acoustic modem [15] to develop a flexible reprogrammable acoutic model with new capabilities suh as reprograrcmability, ranging. TDMLd corcornunisations, and slf-spnchorization.

\section{THE UNDERWMTER SENSOR NETWORK}

A. Handwe

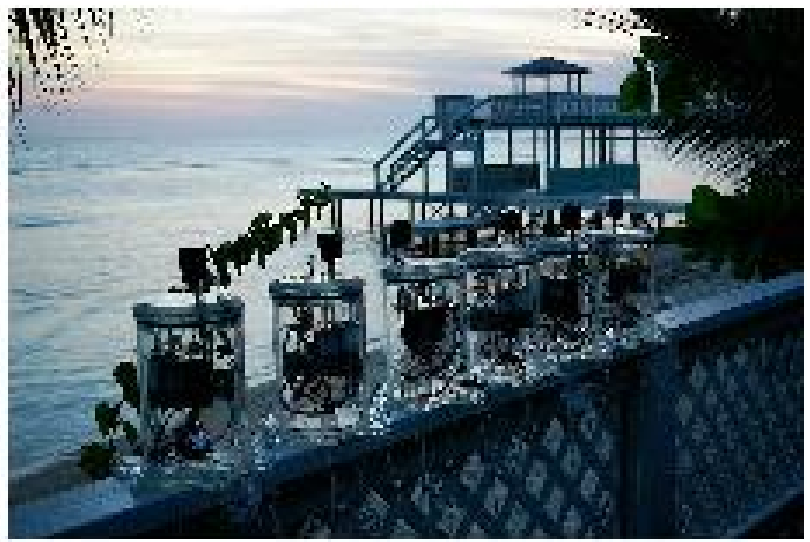

Fig 1. A picture of some sensor nodes dyije.
In our previous work [14] we described our frust underwater sersor returork. This retwork had linited acoustic sapabilities. Builing on this work, we developed a second generation underwater servor network that has retained the original goals and adresed them with a different design and entanced aspabilities.

Phe have built a second generatian underwater sensor nodes alled A gavindes (see Figure 1). So far we have built 10 rodes. Each node is build around a CPU unit, based on the ATruega128 procesor, with 128 kbyte of program flash meroory, 4 kby te of RdM, and 512kby te of flash mernory for data logging/storage. This board has tercperature and pressure ersons on it and irquts for 6 other 24bit analog or digital sensons. The underwater servor node is contaired in an acrylis watertight $\varphi$ linutrical container with a ratius on $15 \mathrm{am}$ and height of $25 \mathrm{am}$ The caps of the tube ane roolded to fit the electronics that need to be there (e.g. the optical receiver and tramanitter, the acoustic transaber, and eables). The bottom eap of the box has a winged system that allows the adition of freestanuing reasuring devices and prorides a suspersion mecharism for weights.

The rother board is interfaced to a special optical conornmications boand through a serial link. The optical board has its oun processor and uses $532 \mathrm{~nm}$ light. It is capable of a rarge of $2.2 \mathrm{~m} / \mathrm{gm} \mathrm{m}^{1}$, within a cone of $90 / 30$ degres and a maxirmm data rate of $320 \mathrm{cbit} / \mathrm{s}$. Aditiomally, there is an acoustic concroulrivation module using $30 \mathrm{kHz}$ FSK modulation and an in-house built trarodwer with a rarge tested u to $400 \mathrm{~m}$ (we believe it can go farther) and a dats rate of 300 bits/s.

For sensing, each node has a pressure seroor, ternperatine ervor, and a $640 \times 490$ color camera. The top side of the finst generation sersor bax contained a 1 to ran rod with an LED beacon. The rod was ued by an dUV to looste the box, dook, and piak it up. Futune versions of the second generation sereor node will contain swh a dosking rod. In adition, the node will contain a XENON flash tube for increasing the distance for reliable rode losation to about 20 reters. The servor rode is powered by $72 \mathrm{amp} / \mathrm{hour}$ Lithium Ion batteries. When all the comporents of the rode run at full power (e.g. the comoromication hardware is fully powered and operates contimuonsly and the all sensons ane aloo fully powered and sample

\footnotetext{
${ }^{1}$ The $8 \mathrm{~m}$ renge requines lanes on ore of the devices and ictively pointing it towerd to other
} 
continuously) the battery provides 2 weeks of continuous operation. In sleep mode the battery provides 1 year of continuous usage. The box is weighted to be $20 \%$ negatively buoyant, and balanced such that if dropped in water it always lands top up.

The sensor node design has been planned for operations up to $200 \mathrm{~m}$ of depth and has been tested in a pressure chamber up to $50 \mathrm{~m}$. We have deployed the nodes in different ocean, lake and river environments up to $10 \mathrm{~m}$. The sensor node is deployed manually (by throwing overboard) or it can be deployed with high precision using a robot. The node has lines of manually adjustable length. The nodes stays suspended with weights attached to the line at the bottom of the water basin.

\section{B. Capabilities}

The sensor system was built in such a way that the system is very easy to deploy. The nodes can just be placed in the water and they will automatically localize themselves using ranges obtained from the acoustic modems. Before the nodes can localize themselves they must decide on a communication schedule. To do this we use a self-synchronizing time division multiple access (TDMA) scheme to schedule messages (see Section III).

The static localization algorithm is a robust localization algorithm we developed based on a $2 \mathrm{D}$ distributed localization algorithm developed in [13]. This algorithm requires the set of ranges between the sensor nodes as input.

The ranges are obtained using the acoustic modems (see Section III.) The ranges are obtained using two different methods. The first is to measure the round trip time of a message between a pair of nodes. This give us a ranges with an accuracy of \pm 3 $\mathrm{cm}$.

The second method is to synchronize the clocks on the nodes and then use a schedule to determine when each node should send a message. The modems have temperature compensated oscillators with about one part per million drift which allows sub-meter ranging accuracy for about thirty minutes before the clocks need to be synchronized again.

In both of these cases we are only able to obtain a single range measurement at any one time. In the case where the ranging is taking place on a moving node such as the robot, this implies that between each range measurement the robot will have moved and this movement needs to be compensated for. We are currently able to obtain a single range measurement every one to four seconds.

\section{Communication}

In this section we describe the acoustic protocols that give our underwater sensor network nodes the capabilities described in Section II-B.

The sensor nodes are networked dually. Optical communication allows line-of-sight fast data transfers as described in [14]. The data rate is $320 \mathrm{~Kb} / \mathrm{s}$ and the nodes. Direct line of site within a 90 degree cone at $2 \mathrm{~m}$ (extensible to $8 \mathrm{~m}$ using lenses) is required for optical communication.

The acoustic communication enables broadcast at lower data rates of $330 \mathrm{kbits} / \mathrm{s}$ at distances of over $400 \mathrm{~m}^{2}$. In this section we describe the details of the acoustic communication system.

\section{A. Acoustic Modem Hardware}

The acoustic modem is built around a Analog Device Blackfin BF533 fixed point DSP processor running at $600 \mathrm{Mhz}$. For transmition the processor generate a PWM signal which is amplified by a $10 \mathrm{~W}$ D class amplifier, operating at about 90transducer developed in house. For reception, the signal from the same transducer is passed through a band pass filter, and variable gain amplifier which drives a 12 bit A/D sampling the signal at $250 \mathrm{k}$ samples per second.

The modem uses a FSK modulation on a $30 \mathrm{Khz}$ carrier frequency. The symbol size is $3 \mathrm{~ms}$, consisting of $1 \mathrm{~ms}$ transmition and $2 \mathrm{~ms}$ pause. The numbers were determined experimentally. We aimed to reach a good trade off between inter-symbol interference and frequency resolution on the receiver side. Each data packet consists of a synchronization pulse followed by 16 bytes of data. The synchronization pulse is a linear frequency sweep of $5 \mathrm{~ms}$ recognized on the receiver side by a matched filter. The data consists of 10 bytes of payload, 2 byte CRC, 1 byte for the source ID, 1 byte for the destination ID, 1 byte for the packet type and 1 byte for the slot number (used by the TDMA scheduler).

\section{B. A TDMA Protocol}

A typical network deployment starts with initializing the number of slots at deployment type. Typically, we start with the a number of slots equal to the number of nodes in the system. We can add slots on the fly or at any time over the duration of the network. The additional slots are allocated to support more frequent communication for the moving

\footnotetext{
${ }^{2}$ We have only tested the system up to $400 \mathrm{~m}$ but we believe the range is greater and have plans to test this in the near future.
} 
nodes or for base stations. Nodes are alboated a sot rumber at the beginuing of deployment. However, ary rode can send a comornand to another node to relesse its time slot.

Our TDML protocol uses 4 s time slote. Each time slot is divided into a 2 s master pasket for the dot, owrer, and 2 s resporse time. The resporse time may be one of two categories based on the owner's request. The owner may request comornmisation to a single rode, or canuromisistion to roultiple nodes. In the case of corromurication to a ingle specific rode, the reporse indudes data. However, the reponse pasket is also used to conculte the rarge between the two nodes (e.g, the roundtrip time oftset by 2 sec.) In the case of roultiple destinations the responses are spaced at $200 \mathrm{~ms}$ intervals and are used primarily to compute roultiple ranges within one comarcumication slot. This comroumication roodality does not support rouch data transfer, but it enables a very effivient way of estimating roultiple ranges. This feature is ircportant for applications that use the servor retwork as an external bolization system to localize and trask a moving rode, for example using the algorithm in [t] , as well as for applications that require the network to self-localize and establizh a system of coondinates. for exarcle using the algorithm extersions of [13].

In our TDML implementation, a node can our arpwhere between 0 and $N$ tirce sbots. The owners of the slote can be ohanged drnamically in real time.

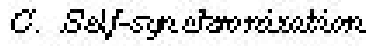

A key featine of our seroor retwrowk system is the ability of the system to self-synchrorize without access to an external abok sounce such as a GFS or to very high-preaision aboks.

The self-symchromization algonithm works as follows. The nodes ane initialized with the total number of sots and each rode lnows its slot number.

When node $n$ with albosted slot $N$ is deploped in water, it waits to hear a conrect master packet for $N \times 4$ s. If the node hears a ressage in this interval, it decodes the slot rumber from the ressage and computes the its own tirce to talk based on this slot rumber. so for exarcple if $N=4$ and is hears a message from the rode whose allocated slot rumber is 3 node $n$ lonows that its time to concromicate is 4 s later. This method allows rodes to synchuorize their internal dook.

If rode $r$ allocated to sbt, $N$ does rot hear arybody during its fust $N \times 4 s n$ starts trancenitiving messages. Evertually ore of the rodes will start comornmisating fint and all the other rodes will spnchorize to it. A possible deatlodk happens if all the nodes start talking at once. This is a lowprobability event. During over 100 trials, we have never encoumtered this situation. We can reduce this probability to a very small value if ore of the nodes is alloosted two time slots.

Once the rodes synchonize upon depbyments they contimue to traramit during their own time slots Every time a rode hears a master padket with correct GRS, the rode respruhuronizes dodks. This procedure loeps the retwork synchuonized over time, and erables nobutrues to dook drifts.

\section{TY. MORIE NODES}

The urderwater sensor network syports mobile nodes such as our underwater nobot called the Autoromous Modular Optical Underwater Robot, (AMOUR), shown in Figne 2. The robot is 157kg. with a maximum speed of $1.5 \mathrm{~m} / \mathrm{s}$. It has a battery life of 8 houns.

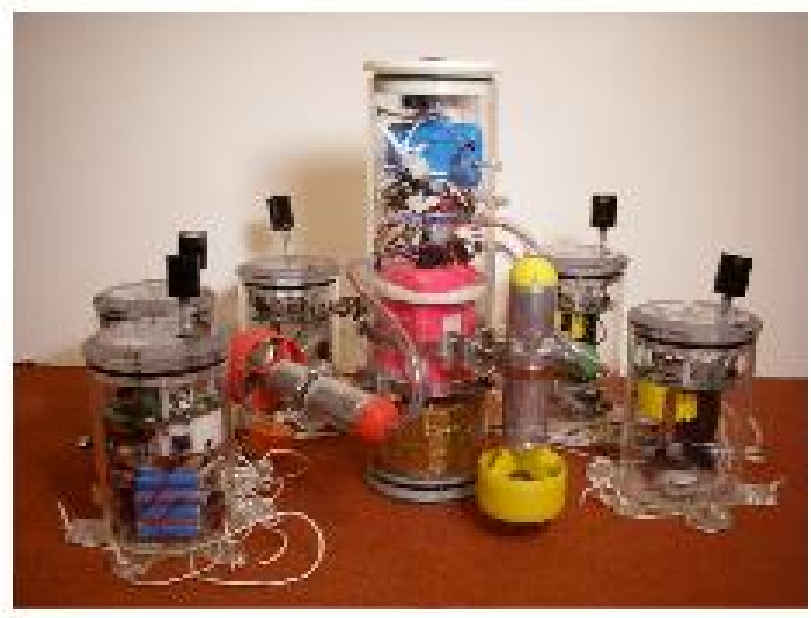

Fig 2. $A$ picture of AMOUR and some sanor node.

The robot has all of the capabilities of the sersor bokes as well as a mone advanced camera system for ue in lowal obetack aroidance. One of the design goals of the robot was to be irexpersive, $x$ it does not have an expersive irertial measnement urit (DMU). Irstead we rely heavily $a$ the range measnerents we obtain to the seroor rodes to detemine its trajectony through the water

The job of the robot is to travel arourd, dowrload datis from the senor nodes op tically and relocate the sensor rodes. Additionally it gives the network drnamic sarcpling capabilities. If an evertis happening of interest the robot ean rove to that, area to provide 
denser sensor sampling. We envision having many robots in the final system to provide highly dynamic sampling and faster download of the data from the static nodes.

To be able to find the sensor nodes the robot must know precisely where it is at all times. A passive localization and tracking algorithm we developed [7] has been implemented on this sensor network system and used to localize and track the moving robot.

\section{EXPERIMENTS}

We have deployed the sensor nodes and the robot in the ocean (Moorea, French Polynesia), in the river (Charles River, MA) and in a lake (Otsego, NY) and collected extensive networking and localization data for this system. We have done over 100 experiments with the sensor network. Typical data from such deployments in shown in Figures 4, 3, and 5 . These figures show the performance of a four node deployment.

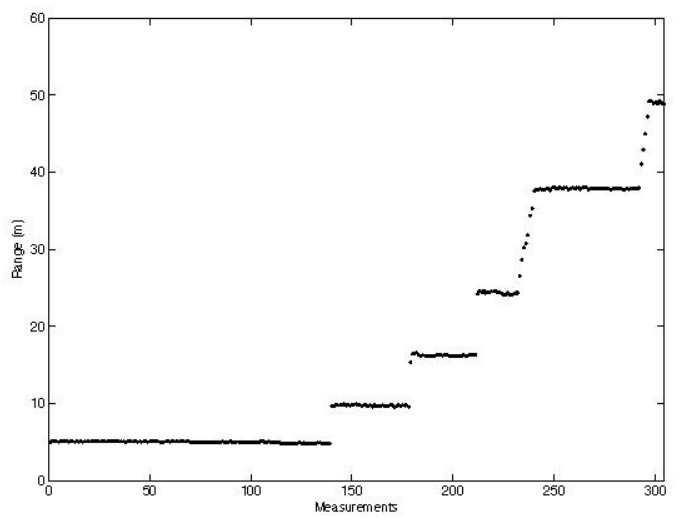

Fig. 3. The ranges computed between a fixed sensor node and a moving sensor node in meters. The $\mathrm{x}$-axis shows the measurement number. The y-axis shows the estimated distance. We have performed tests at 4 different distances: $4.75 \mathrm{~m}, 9.67$ $\mathrm{m}, 16.15 \mathrm{~m}$, and $24.7 \mathrm{~m}$. The $\mathrm{y}$ values show the sensor network estimates.

Figure 3 shows the ranges computed between a fixed sensor node anchored in a lake at a depth of $3 \mathrm{~m}$ to a sensor network node moved in a straight line by the underwater robot. The first set of measurements were taken at a distance of $4.75 \mathrm{~m}$. The average sensor network node estimated the range at 5.01 $\mathrm{m}$. The second set of measurements were taken at $9.67 \mathrm{~m}$. The average estimated range was $9.72 \mathrm{~m}$. The third set of measurements was taken at $16.15 \mathrm{~m}$. The average estimated range by the network was $16.19 \mathrm{~m}$.

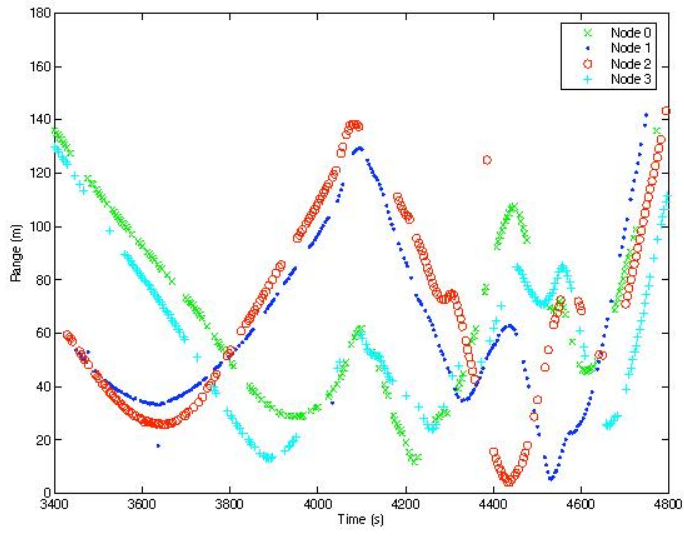

Fig. 4. The ranges computed by four sensor network nodes to a moving node over time. The $\mathrm{x}$-axis shows time. The $\mathrm{y}$-axis shows distance in meters.

The fourth set of measurements was taken at $24.7 \mathrm{~m}$. The average estimate by the sensor network node was $24.35 \mathrm{~m}$.

Figure 4 shows the ranges to a moving node (the robot) computed by a sensor network of 4 nodes. In this experiment the robot was commanded to move in the field of sensor nodes. The gaps in the graph denote communications that were not successful. Figure 5 shows the details of the communication success. For sensor network node $1,58 \%$ of the messages were received correctly. In the case of node $2,64 \%$ of the messages were received correctly. For node $364 \%$ of the messages were received correctly. Finally, for node 4 the communication success rate was $50 \%$. The message loss was due to changing water conditions and lake bottom profile as the moving node traveled across the lake. We need to understand better how the lake geography and composition affects acoustic communication. We believe that a success rate of over $50 \%$ is sufficient for the type of monitoring and tracking applications we wish to perform with this system.

\section{Conclusion}

In this paper we described the hardware and communications support for an underwater sensor network designed and implemented in our lab. Our experiments show that this is a capable and usable platform for water applications in shallow waters at depths less than $100 \mathrm{~m}$. We are especially interested in using this system to provide automated data collection for marine biology applications related to 


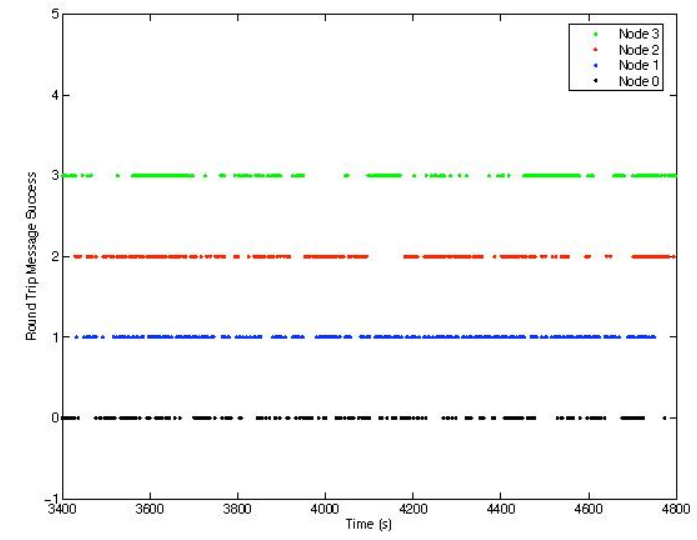

Fig. 5. Communication success for 4 sensor network nodes communicating to a moving node networked acoustically to the system. The communication for each node is displayed as a line. Gaps correspond to lost messages.

understanding and modeling coral reefs. This sensor network could sustain operations at greater depths by replacing the acrylic enclosure with a glass or titanium enclosure.

\section{ACKNOWLEDGEMENTS}

We are grateful to the NSF and ONR (under the PLUS-Net project) for supporting in part this research.

\section{REFERENCES}

[1] Autonomous Ocean Sampling Network (AOSN)II, collab-

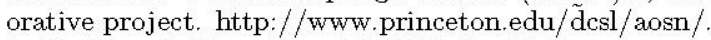

[2] I. Akyiditz, D. Pompli, and T. Melodia. Underwater sensor networks: research challenges. Ad-hoc Networks, 2005 (to appear).

[3] K. Audenaert, H. Peremans, Y. Kawahara, and J. Van Campenhout. Accurate ranging of multiple objects using ultrasonic sensors. In Proc. IEEE Int. Conf. Robotics and Automation, pages 1733-1738, Nice, France, May 1992.

[4] A. B. Baggeroer. Acoustic telemetry - an overview. IEEE J. Ocean Engineering, OE-9(4):229-235, October 1984.

[5] J. G. Bellingham. In E. Bovio and R. Tyce, editors, Autonomous Underwater Vehicle and Ocean Modelling Networks: GOATS 2000 Conference Proceedings. NATO SACLANT Undersea Research Centre, La Spezia, Italy, 2000.

[6] T. Curtin, J. G. Bellingham, J. Catipovic, and D. Webb. Autonomous ocean sampling networks. Oceanography, $6(3): 86-94,1993$.

[7] Carrick Detweiler, John Leonard, Daniela Rus, and Seth Teller. Passive mobile robot localization within a fixed beacon field. In Proc. of the 2006 International Workshop on Algorithmic Foundations of Robotics, New York, August 2006. Springer-Verlag.
[8] M. Dunbabin, I. Vasilescu, P. Corke, and D. Rus. Data muling over underwater wireless sensor networks using an autonomous underwater vehicle. In Proceedings of the 2006 International Conference on Robotics and Automations, Orlando, FL, May 2006

[9] E. Fiorelli, N. E. Leonard, P. Bhatta, D. Paley, R. Bachmayer, and D. M. Fratantoni. Multi-AUV control and adaptive sampling in Monterey Bay. In Proceedings of the IEEE Autonomous Underwater Vehicles: Workshop on Multiple AUV Operations, Sebasco, ME, USA, June 2004.

[10] Heidemann, J., LI, Y., SYed, A., Wills, J., AND Ye, W. Underwater sensor networking: Research challenges and potential applications. Tech. Rep. ISI-TR-2005-603, USC/Information Sciences Institute, July 2005.

[11] Hill, J., Bounadonna, P., And Culler, D. Active message communication for tiny network sensors. In $I N$ FOCOM (2001).

[12] Howe, B. M., McGinnis, T., and Kirkham, H. Sensor Networks for Cabled Ccean Observatories. EGS - AGU EUG Joint Assembly, Abstracts from the meeting held in Nice, France, 6 - 11 April 2003, abstract \#12598 (Apr. 2003), 12598-+.

[13] David Moore, John Leonard, Daniela Rus, and Seth Teller. Robust distributed network localization with noisy range measurements. In Proc. 2nd ACM SenSys, pages 50-61, Baltimore, MD, November 2004.

[14] I. Vasilescu, K. Kotay, D. Rus, P. Corke, and M. Dunbabin. Data collection, storage and retrieval with an underwater sensor network. In ACM Sensys, 2005.

[15] Whoi modem: http://acomms.whoi.edu/micromodem/. 